\title{
Corneal Biomechanical Changes after Collagen Cross- Linking from Porcine Eye Inflation Experiments
}

\author{
Sabine Kling, ${ }^{1}$ Laura Remon, ${ }^{1}$ Alfonso Pérez-Escudero ${ }^{1}$, Jesus Merayo-Lloves, ${ }^{2}$ and \\ Susana Marcos ${ }^{1}$
}

Purpose. Understanding corneal biomechanics is important to refractive or therapeutic corneal treatments. The authors studied the corneal response to variable intraocular pressure (IOP) in porcines eyes after UV collagen cross-linking (CXL), in comparison with untreated eyes.

Methods. Twenty-three enucleated eyes were treated with standard CXL conditions ( $365 \mathrm{~nm}, 3 \mathrm{~mW}, 30$ minutes), and 15 contralateral eyes served as control. Eyes (within a humidityand temperature-monitored wet chamber) were measured by Scheimpflug corneal three-dimensional topographer. Images were obtained automatically while IOP either remained constant ( 14 eyes) or increased ( 24 eyes) by $40 \mathrm{~mm} \mathrm{Hg}$ and then decreased (4-mm Hg steps). Measurements were performed immediately after treatment and 24 hours later. Corneal geometry was analyzed as a function of IOP, and whole globe stress-strain curves were calculated.

RESUlTs. Instillation of riboflavin-dextran solution reduced corneal thickness (by $281 \pm 5 \mu \mathrm{m}$ ). Cross-linking produced a $1.54 \times$ reduction in corneal thinning and $2.8 \times$ reduction in corneal apical rise with increased IOP. Anterior and posterior cornea flattened with increased IOP (less flattening in CXL eyes) and became steeper with decreased IOP. The horizontal meridian flattened significantly $(P<0.01)$ more than the vertical meridian. Young's modulus was higher in cross-linked eyes $\left(1.096 \pm 0.30 \mathrm{kN} / \mathrm{m}^{2}\right)$ than in non-cross-linked eyes $\left(0.692 \pm 0.30 \mathrm{kN} / \mathrm{m}^{2}\right)$. Hysteresis in nontreated eyes was also larger than in cross-linked eyes.

Conclusions. Cross-linking stiffened porcine corneas significantly. Both experimental data and stress-strain analysis are valuable for finite element models to improve understanding of CXL and its predictability. Although differences are expected between human corneas in vivo and porcine corneas ex vivo,

From the ${ }^{1}$ Instituto de Optica, Daza de Valdés, Consejo Superior de Investigaciones Científicas, Madrid, Spain; and the ${ }^{2}$ Instituto Universitario de Oftalmobiología Aplicada, Universidad de Valladolid, Valladolid, Spain.

Presented in part at the annual meeting of the Association for Research in Vision and Ophthalmology, Fort Lauderdale, Florida, May 2009 .

Supported by Ministerio de Ciencia e Innovación Grant FIS200802065 and EUROHORCS-ESF Grant EURYI-05-102-ES (SM); Ministerio de Ciencia e Innovación FPU predoctoral fellowship (APE); CSIC JAEProgram (LR); Ministerio de Ciencia e Innovación Erasmus Program and FPI predoctoral fellowship (SK); Unidad Asociada Instituto de Optica-CSIC/Instituto de Oftalmobiología Aplicada-Universidad de Valladolid; and IROC Medical (SK).

Submitted for publication August 25, 2009; revised November 25, 2009, and January 4, 2010; accepted March 7, 2010.

Disclosure: S. Kling, None; L. Remon, None; A. Pérez-Escudero, None; J. Merayo-Lloves, None; S. Marcos, None

Corresponding author: Susana Marcos, Instituto de Óptica, Daza de Valdés, Consejo Superior de Investigaciones Científicas, Serrano 121, 28006 Madrid, Spain; susana@io.cfmac.csic.es. the results are consistent with clinical data found in patients. The apparent biomechanical anisotropy of pig corneas must be confirmed in humans. (Invest Ophthalmol Vis Sci. 2010;51: 3961-3968) DOI:10.1167/iovs.09-4536

Corneal collagen cross-linking [CXL] is an emerging tech$\checkmark$ nique aimed at strengthening corneal tissue. It has been successful in slowing down the progression of keratoconus and preventing ectasia. The technique works through the application of riboflavin (vitamin B2) as a photosensitizer (after epithelium removal) and irradiation of the cornea with UV-A to increase the formation of intrafibrillar and interfibrillar covalent bonds by photosensitized oxidation. ${ }^{1,2}$ After it was first proposed by Seiler $^{3}$ in 1998 , several studies have examined corneal CXL with regard to dosage parameters of riboflavin, safety of UV-A radiation, ${ }^{4-6}$ penetration depth, ${ }^{7}$ and efficacy of the treatment in keratoconus ${ }^{8}$ or of the combined effect of corneal surgical treatments (i.e., intracorneal rings, laser refractive surgery, or orthokeratoplasty). ${ }^{9,10}$

The formation of additional cross-links between individual collagen molecules leads to an increase in corneal rigidity. Biomechanical changes have been demonstrated in human, 8,11 porcine $^{11}$ and rabbit ${ }^{12,13}$ corneas. To a large extent, these studies rely on static stress-strain experiments on corneal strips. A recent study reported a significant increase in corneal rigidity after cross-linking, indicated by a rise in stress in treated porcine corneas (by 71.9\%) and human corneas (by 328.9\%) and in Young's modulus by a factor of 1.8 in porcine corneas and of 4.5 in human corneas ${ }^{14}$ using extensometry methods.

Previous studies have stressed the limitations of corneal strip extensometry measurements to measure biomechanical properties. ${ }^{15}$ Some limitations are that strip specimens originate from a curved sample, that the corneal structure is disrupted because the lamellae are cut, and that several crucial constraints are ignored (real pachymetry or meridional differences, among others). Although some models have been developed to improve the accuracy of extensometry tests, ${ }^{15}$ it has been suggested that the resultant material stress-strain relationship from strip extensometry is considerably stiffer than that derived using inflation tests, and the differences between the reported estimates of Young's modulus using one or the other technique can vary by an order of magnitude.

Whole eye globe inflation testing overcomes many of the problems of extensometry, although care must be taken to control tissue hydration if the results aim to mimic in vivo biomechanical responses. Inflation testing methods have been used for many years to derive ocular rigidity ${ }^{16-18}$ and to determine pressure-displacement response relationships in the cornea. ${ }^{15,18-20}$ Some studies ${ }^{20,21}$ use only the anterior portion of the globe (cornea and a ring of sclera) attached to a chamber with a complex fixture scheme to separate corneal deformation from scleral deformation. Although this technique has the advantage of avoiding the potential influence of the supporting sclera in the measurements, the use of the intact eye represents 
a closer situation of the in vivo response than a mechanical fixation of the sclera, because fixation at the limbus could introduce a nonphysiological boundary condition that could affect central corneal strains. ${ }^{22}$ Given that the Young modulus of the sclera is 3 to 3.5 times higher than the cornea, ${ }^{17}$ the impact of the scleral biomechanical properties on the corneal deformation measurements is potentially small. In the whole eye model, the limbal junction is allowed to move, which is thought to be crucial to establish a biomechanical model mimicking the optical function of a real eye. ${ }^{18,23-25}$

Porcine models are frequently used to assess biomechanical properties that may be extrapolated to human corneas. Despite differences in corneal thickness (the average porcine cornea is $1.6 \times$ thicker than the human cornea) and stiffness (the porcine cornea is more elastic than the human cornea), ${ }^{26,27}$ it is accepted that the porcine cornea is a suitable model for the human cornea in mechanical properties. They both exhibit similar stress-strain behavior under short- and long-term loading and react similarly to sustained loading, although the viscoelastic creep is higher in human than in porcine corneas. Corneal strip extensometry experiments on human and porcine cross-linked corneas reveal a larger stiffening effect on human than on porcine corneas, which has been attributed to the relatively larger corneal cross-section irradiated in the overall thinner human cornea. ${ }^{14}$

To our knowledge, this study presents for the first time inflation tests on porcine corneas that had undergone CXL treatment compared with non-cross-linked corneas. In addition, though most inflation experiments only report the shift of the corneal apex in response to the variation of intraocular pressure, we also report measurements of anterior and posterior corneal shape and thickness as a function of increased and decreased pressure. These results allow us to accomplish direct comparisons with clinical refractive changes measured in vivo. In addition, these measurements can be used to derive estimates of stress-strain behavior and Young's modulus after cross-linking (and to estimate the changes of corneal stiffness and biomechanical properties induced by the treatment). The full parametric characterization of corneal deformation as a function of pressure will be valuable to improve the predictability of finite element modeling of the cornea and ultimately the predictability of the procedure.

\section{Materials ANd Methods}

\section{Eyes}

Measurements were performed on fresh enucleated porcine eyes from a local slaughterhouse. A total of 38 eyes were used in the study. Twenty-three eyes were treated with cross-linking. Control eyes (15 contralateral eyes from the treated eye) were not irradiated with UV light but underwent epithelium removal and riboflavin instillation. Sixteen cross-linked eyes and eight control eyes were measured under varying intraocular pressure, and seven cross-linked and seven control eyes were measured under constant pressure.

Measurements were performed within 4 hours of death (immediately after treatment) and were repeated 24 hours later. Between measurements the eyes were kept in a refrigerator at $4^{\circ} \mathrm{C}$ in a chamber with cotton soaked in physiological saline (sodium chloride $0.9 \%$ ).

\section{Cross-linking Treatment}

CXL treatment was performed (UV-X radiation system; IROC, Zurich, Switzerland) on 23 enucleated porcine eyes in accordance with standard clinical procedures. ${ }^{1}$

First, the whole epithelium was removed with a scalpel to allow the photosensitizer solution to diffuse into the stroma and to facilitate UV light exposure. Then a solution containing $0.125 \%$ riboflavin (vitamin B12) and $20 \%$ dextran was instilled every 3 minutes for 30 minutes

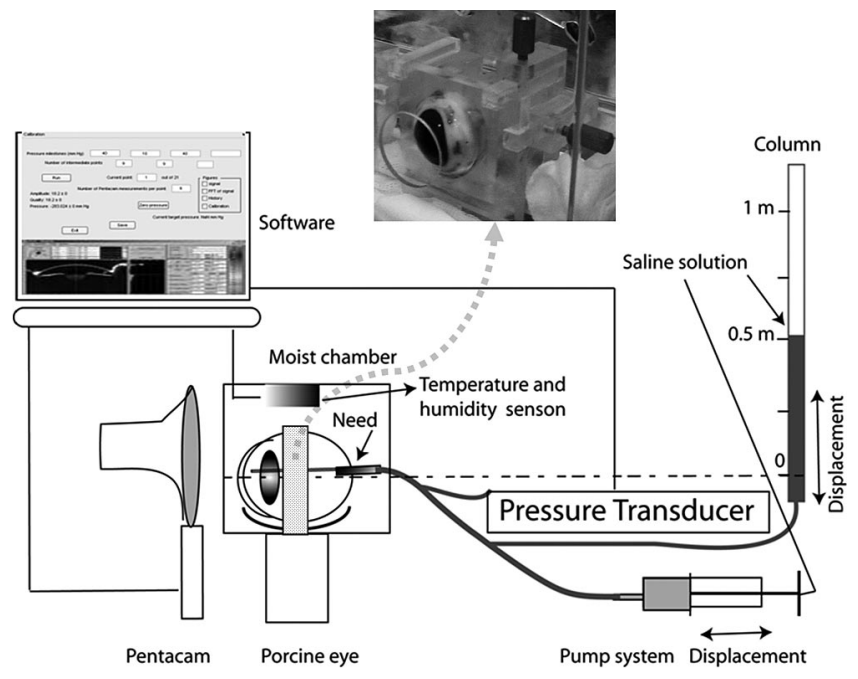

FigURE 1. Schematic setup of the ocular inflation test.

before irradiation to increase the efficiency of the irradiation and to avoid light damage to the retina. The corneas were then irradiated with UV-A $(365 \mathrm{~nm})$ for 30 minutes (while riboflavin was instilled every 3 minutes during irradiation) with an irradiance of $3 \mathrm{~mW} / \mathrm{cm}^{2}$. The UV-A delivery system was located $60 \mathrm{~mm}$ from the cornea. We used an irradiation area of 10-mm diameter (large) and 6-mm diameter (small).

\section{Scheimpflug Imaging}

We used a Scheimpflug-based camera (Pentacam; Oculus GmbH, Wetzlar, Germany) anterior segment imaging system based on the Scheimpflug principle. The Scheimpflug camera is a modification of a slit-lamp camera, with a modified geometry to improve depth of focus. The slit rotates around the optical axis of the instrument, allowing the capture of 25 anterior segment diametral sections at different angles and resulting in three-dimensional elevation maps of the anterior and posterior corneal surfaces. In a previous study, we demonstrated quantitative anterior segment biometry and cornea and crystalline lens geometry after application of custom-developed optical and geometric distortion-correction algorithms on Scheimpflug camera (Pentacam; Oculus $\mathrm{GmbH}$ ) images. ${ }^{28}$ In addition, we validated the accuracy of the measurements of the anterior and posterior corneal surface geometry provided by the commercial system with the use of a new hybrid porcine/plastic eye model. We demonstrated that measurements of the posterior corneal radius of curvature and asphericity are not affected by changes in the anterior corneal surface, ${ }^{29}$ and we found that the used Scheimpflug system (Pentacam) provides a good posterior radius. Therefore, the system can be used reliably to estimate anterior and posterior corneal deformation by intraocular pressure changes.

Measurements of vertical and horizontal radii of curvature and asphericity were obtained by fitting the raw elevation maps provided by the software to biconic surfaces, using custom routines written in computing software (MatLab; MathWorks, Natick, MA). ${ }^{29,30}$ Central pachymetry was obtained directly from the commercial software. The axial shift of the corneal apex was estimated by measuring horizontal and diagonal distance from the apex to a constant reference point in Scheimpflug images (white dot in the upper left corner). These data were used to calculate the apical displacement on a subset of eyes (four untreated eyes and four cross-linked eyes), which were not moved at all during the measurements.

\section{Experimental Setup}

Figure 1 shows a diagram of the experimental setup used to measure corneal deformation on application of variable intraocular pressure. Intraocular pressure was monitored by means of a water column and a pressure transducer and was modified by infusion of saline solution 
into the eye through a needle automatically controlled by a pumping system (NE-500; New Era Pump Systems, Inc., Wantagh, NY). The humidity and temperature in the moist chamber in which the eyes were placed during the measurements were also continuously monitored and recorded.

Sinusoidal alternating voltage was achieved by constant playing of a 440-Hz tone from a personal computer sound card amplified to serve as voltage supply for a Wheatstone bridge setup. The pressure sensor was taken as the variable resistance that measured the current pressure of the water column. Finally, the difference of voltage was measured, amplified again, and fed back onto the microphone entrance of the computer. In addition, the Scheimpflug system data acquisition was automatized (by automatic control of the keyboard) so that threedimensional anterior segment images were also recorded without the need of manual control. The system was controlled by dedicated software written in computing software (MatLab; MathWorks).

Intraocular pressure was increased from 18.5 to $58.5 \mathrm{~mm} \mathrm{Hg}$ and then was decreased to $18.5 \mathrm{~mm} \mathrm{Hg}$. Scheimpflug measurements were taken at $4-\mathrm{mm} \mathrm{Hg}$ increments of the IOP increases, as controlled by the pressure monitoring system or every 5 minutes (in the experiments in which the IOP was kept constant at $19 \mathrm{~mm} \mathrm{Hg}$ ). Temperature and humidity were recorded every 2 seconds throughout the experiment.

\section{Experimental Protocols}

The enucleated porcine eyes were placed in a custom-made holder to guarantee proper centration during the application of the treatment and during the measurements. A view of the holder can be seen in Figure 1. The holder consists of two rectangular plastic parts, each with an inwrought half-sphere of $20-\mathrm{mm}$ diameter. By regulating the distance of the plastic parts by means of screws, the holder was allowed to accommodate the individual porcine eyes. The eyes always were placed with the longer side of the cornea in the vertical direction. Compared with the real orientation of the eye in the pig, in the experiment the eye was turned $90^{\circ}$, which allowed a better view and more accurate alignment. (In Results and Discussion, however, we use horizontal and vertical to refer to the actual orientation of the eye in the pig.) The eye holder was placed in a methacrylate box in which wet cotton cloths provided a constantly humid atmosphere. A window at the front of the box allowed an unrestricted view of the eye by the Scheimpflug system.

Saline infusion was produced by introducing a needle through the optical nerve head into the anterior segment of the eye. The needle was connected to an infusion tube to a $\mathrm{NaCl}$ solution. Preliminary measurements were conducted by entering the anterior segment of the eye superiorly, through the sclera, leaving the cornea untouched (Pérez-Escudero A, et al. IOVS 2008;49:ARVO E-Abstract 664) However, this procedure was more challenging, and we did not observe any difference between results.

An entire experimental session on each eye typically took 40 minutes. The initial measurement was taken at $18.5 \mathrm{~mm} \mathrm{Hg}$ and subsequently at $4-\mathrm{mm} \mathrm{Hg}$ step increases. Pressure was increased to $58.5 \mathrm{~mm} \mathrm{Hg}$ and then decreased, at $4-\mathrm{mm} \mathrm{Hg}$ steps, with data also collected sequentially. Sessions on both eyes of the same animal were typically performed consecutively and randomly, with one eye having undergone the cross-linking treatment and the other having undergone only epithelial removal and riboflavin instillation. A set of preliminary measurements was performed simultaneously increasing the pressure in both eyes and taking consecutive images in each eye at identical pressure levels. We did not observe any difference between the results from this procedure and those from automatic full measurements in one eye after the other.

A set of control experiments was performed by keeping the pressure constant at $19 \mathrm{~mm} \mathrm{Hg}$ and taking images at 5-minute intervals during the same period as the variable-pressure experiments. Measurements were also collected in cross-linked and non-cross-linked eyes. Typically, each eye was measured in two identical experimental sessions 24 hours apart

\section{Simple Biomechanical Model: Young's Modulus and Hysteresis}

We have developed a model for the corneal stress-strain relationship based on IOP variation and related changes in thickness and radius of curvature to provide estimates of Young's modulus in the untreated and cross-linked corneas. The model was similar to that used in previous inflation tests, ${ }^{18,19,22,24}$ though fewer assumptions were required because the corneal radius of curvature was measured directly in the experiment (and not necessarily derived from the apical rise, which was the only experimental input data available in other studies ${ }^{15,20}$ ).

The model assumes that corneal thickness and mean radius of curvature are a function of IOP-that is, increasing IOP will make the globe of the eye expand. Corneal thickness decreases because the cornea becomes stretched over a larger area. However, stretching the cornea induces stress that acts to prevent further corneal extension. Both forces-the IOP tendency to stretch the cornea and the stress tendency to prevent it-arrange to form a stable equilibrium. With basic equations for pressure $p=F / A$ (where $p$ represents pressure, $F$ represents force, and $A$ represents surface of eye globe) and strain $\sigma=$ $F / A$ (where $\sigma$ represents stress, $F$ represents force, and $A$ represents corneal cross-sectional area), we can express stress as a function of IOP:

$$
\sigma=\frac{R \cdot p}{2 \cdot d}
$$

where $\sigma$ represents stress; $R$ represents mean radii of anterior, posterior, horizontal, and vertical curvature; $p$ represents IOP; and $d$ represents corneal thickness.

The strain on the stress produced by IOP can be expressed by $\varepsilon=$ $\Delta R / R$, where $\varepsilon$ represents strain, $\Delta R$ represents difference in radius of curvature with respect to an initial measurement, and $R$ represents radius of curvature.

Limitations of the model include the fact that the mechanical properties of the sclera are not considered to contribute to the response of the cornea to pressure elevation because of the high rigidity and low elasticity of the sclera compared with the cornea. ${ }^{17,19}$ of course, this is an approximation because scleral effects cannot be totally neglected. Nevertheless, it appears reasonable as a first estimation of stress-strain curves in whole eye globe experiments. Moreover, the stress-strain calculation refers to a homogenous membrane sphere, with the same elasticity, rigidity, and thickness all over the globe. Therefore, the influence of corneal thickness gradient or local elasticity and rigidity differences cannot be detected. In addition, the cornea is assumed to be purely elastic. The model is based on horizontal/vertical averages of corneal radii changes and does not include potential meridional differences in deformation.

\section{Results}

\section{Changes in Corneal Thickness}

A dramatic decrease in corneal thickness occurred immediately after the application of riboflavin-dextran solution (duration, 30 minutes) to the deepithelialized corneas. Figure 2 shows examples of Scheimpflug images before and after riboflavin application. On average, a decrease of $281 \pm 5 \mu \mathrm{m}$ was found after the application of riboflavin. Another decrease was observed 30 minutes later, after UV illumination, which was higher in control eyes $(88 \mu \mathrm{m})$ than in cross-linked eyes $(31$ $\mu \mathrm{m})$. After riboflavin instillation, the radius of curvature changed by $165 \pm 655 \mu \mathrm{m}$ (anterior) and $-774 \pm 1689 \mu \mathrm{m}$ (posterior).

Figure 3 shows the change in corneal thickness as a function of IOP change (first increased from $18.5 \mathrm{~mm} \mathrm{Hg}$ to 58.5 $\mathrm{mm} \mathrm{Hg}$, then decreased) immediately after treatment (Fig. 3A) and then 24 hours after treatment (Fig. 3B). Results are the 


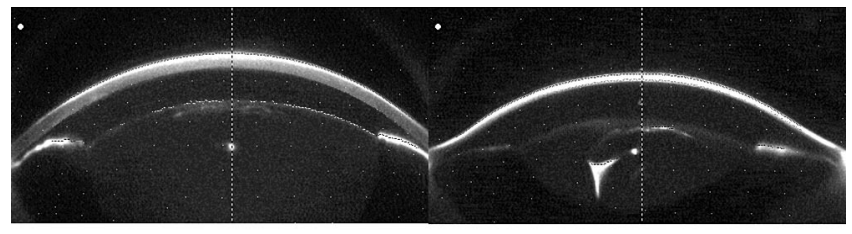

A

B

FIGURE 2. Scheimpflug images of enucleated porcine corneas before (A) and 30 minutes after (B) riboflavin-dextran instillation, immediately after treatment.

average across 16 cross-linked eyes and 8 non-cross-linked eyes. In each case the change is referred to the initial corneal thickness in each eye. Initial corneal thickness varied between 430 and $636 \mu \mathrm{m}$ in the cross-liked eyes and 353 to $647 \mu \mathrm{m}$ in the non-cross-linked eyes immediately after treatment (Fig. 3A) and between 999 and $1221 \mu \mathrm{m}$ in the cross-liked eyes and 945 to $1259 \mu \mathrm{m}$ in the non-cross-linked eyes 24 hours after treatment (Fig. 3B). Immediately after treatment, corneal thickness changes (Fig. 2A) were dominated by hydration effects, as the increase in corneal thickness occurred similarly under variable IOP $(1.03 \mu \mathrm{m} / \mathrm{min}$ [CXL]) and $0.83 \mu \mathrm{m} / \mathrm{min}$ (untreated) or constant IOP $(1.1 \mu \mathrm{m} / \mathrm{min}[\mathrm{CXL}])$ and $2.0 \mu \mathrm{m} / \mathrm{min}$ (untreated). Removal of the epithelium made the corneas more susceptible to hydration; under humid and pressure-controlled conditions in the same moist chamber, we had not observed an increase of corneal thickness at constant IOP in virgin corneas in previous experiments in our laboratory (Kling S, et al. IOVS 2009;50:ARVO E-Abstract 5477). After 24 hours, hydration effects were minor (around $10 \%$ of the values immediately after the treatment, as shown from data under constant IOP) and were dominated by the pressure change. Corneal thickness clearly decreased with the IOP increase and increased with the IOP decrease, with smaller changes in the cross-linked than in the non-cross-linked corneas. A total variation of $40 \mathrm{~mm} \mathrm{Hg}$ in IOP pressure produced decreases in corneal thickness of 51.2
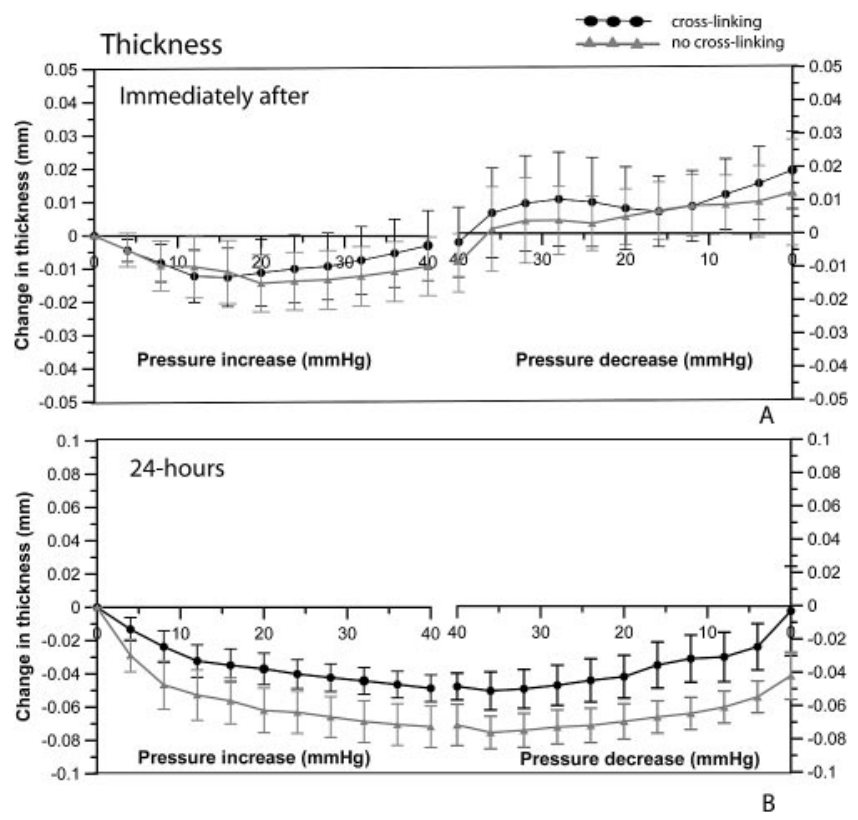

FIGURE 3. Change in corneal thickness with IOP pressure change relative to $18.5 \mathrm{~mm} \mathrm{Hg}$ for cross-linked and non-cross-linked corneas. Symbols represent average data across eyes, and error bars represent SD. (A) Immediately after treatment. (B) 24 hours after treatment. Data are the averages of $16 \mathrm{CXL}$ and 8 non-CXL.
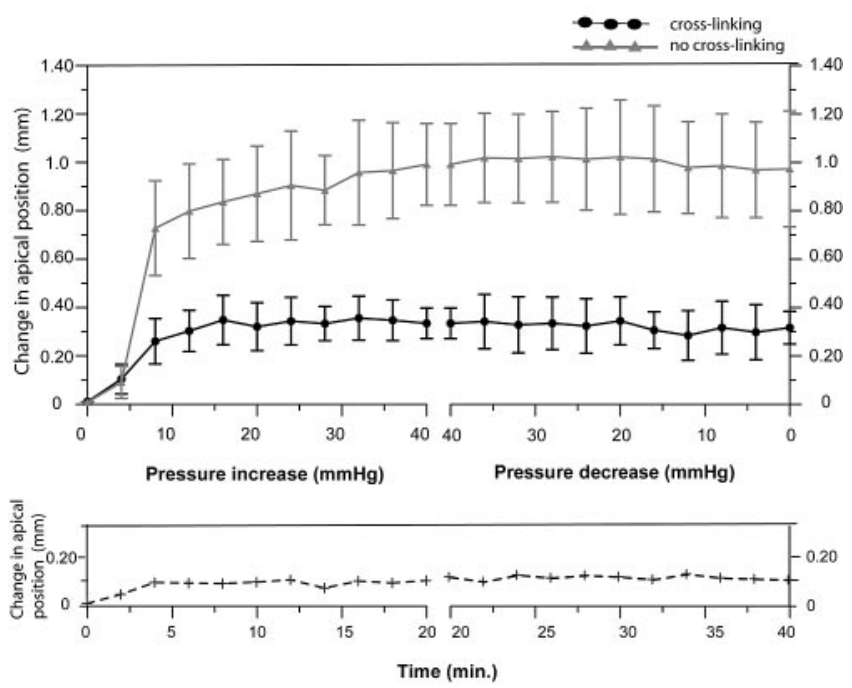

FIGURE 4. 24-hours apex shift as a function of (increasing and decreasing) pressure change relative to $18.5 \mathrm{~mm} \mathrm{Hg}$ for cross-linked and non-cross-linked corneas. Dashed line: apex shift during 40 minutes (equivalent to duration of the pressure variation experiment) under constant pressure. Data are from the period immediately after treatment and are averages of four CXL and four non-CXL. Error bars represent $\mathrm{SD}$.

$\mu \mathrm{m}$ (in cross-linked corneas) and $76.3 \mu \mathrm{m}$ (in non-cross-linked corneas). On average, the changes in non-cross-linked eyes were significantly higher $(1.54 \times)$ than in cross-linked eyes. The differences were statistically significant at all pressure levels $(P<0.01)$. After IOP variations, cross-linked corneas returned to their initial thickness, whereas non-cross-linked corneas remained $42.1 \mu \mathrm{m}$ thinner.

\section{Changes in the Corneal Apex Position}

Figure 4 shows the change in corneal shape position as a function of pressure for cross-linked and non-cross-linked eyes after 24 hours. The apical rise of control eyes that had not undergone pressure changes during the same period is also shown for reference.

There was a significant forward shift in the anterior corneal apex with increasing pressure, which was 2.8 times higher in nontreated corneas $(962 \mu \mathrm{m})$ than in cross-linked corneas $(345$ $\mu \mathrm{m})$. With constant pressure the apex showed only a slight shift and no difference between treated and untreated corneas. Decreasing pressure produced a backward movement of the apex, but it did not return to the initial values.

\section{Change in Vertical and Horizontal Corneal Radius of Curvature}

Immediately after the procedure, we found slight variation in the anterior corneal curvature throughout the session under constant IOP: $-4.5 \mu \mathrm{m} / \mathrm{min}$ (CXL), $4.4 \mu \mathrm{m} / \mathrm{min}$ (non-CXL). The variation was even smaller under constant IOP 24 hours after treatment $(-0.63 \mu \mathrm{m} / \mathrm{min}$ [CXL], $-2.5 \mu \mathrm{m} / \mathrm{min}$ [untreated]).

Figures 5 and 6 show changes of the anterior (Fig. 5) and posterior (Fig. 6) corneal radii of curvature in the vertical meridian with IOP variation immediately after treatment (Figs. $5 \mathrm{~A}, 6 \mathrm{~A}$ ) and 24 hours after the treatment (Figs. 5B, 6B).

Changes in the anterior corneal radius with pressure, and the effect of treatment, were minimal immediately after treatment. Measurements 24 hours after treatment (Figs. 5A, 6B) showed statistically significant differences between crosslinked and non-cross-linked eyes. The radius of curvature 

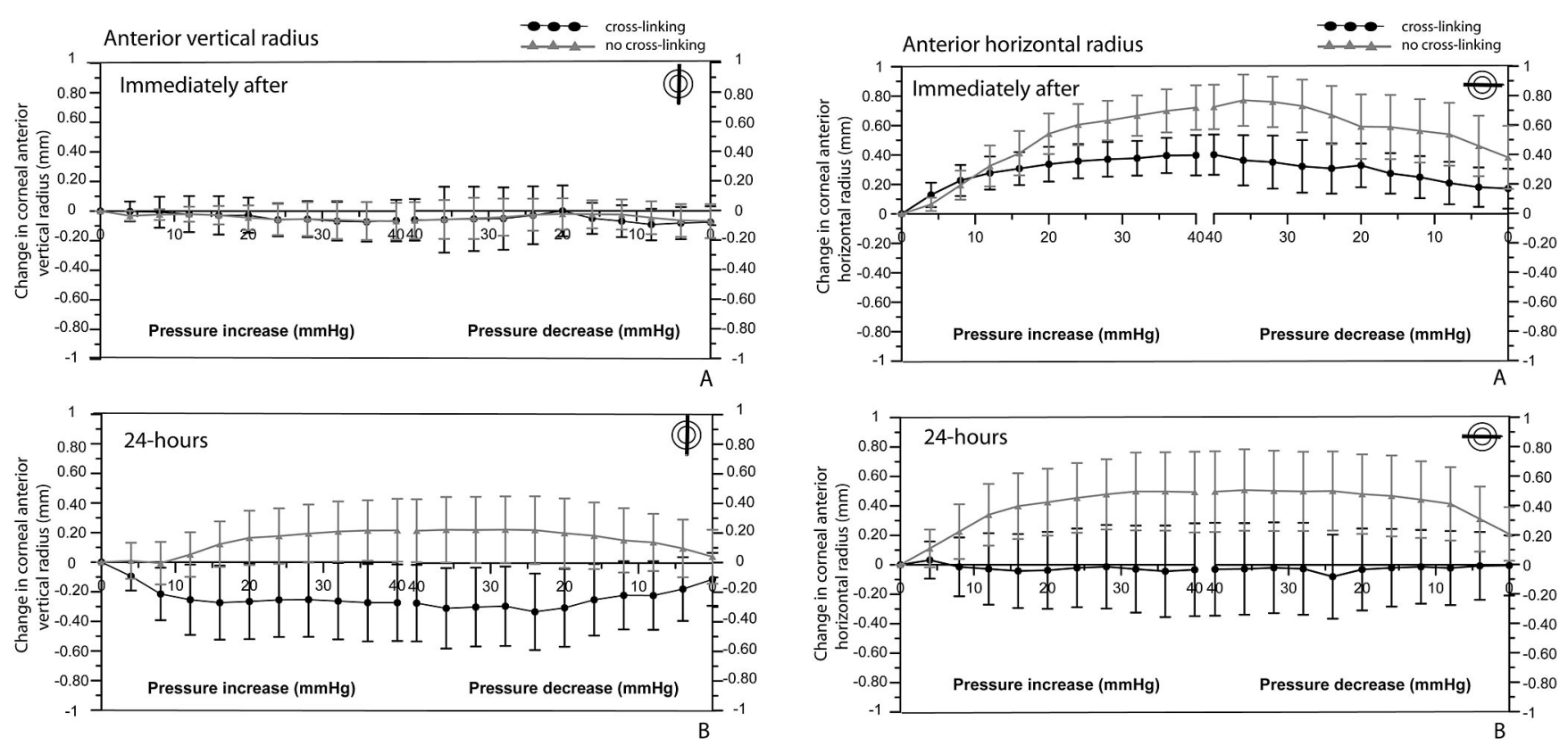

FigURE 5. Change in anterior vertical radius of curvature with varying pressure (relative to $18.5 \mathrm{~mm} \mathrm{Hg}$ ) for cross-linked and non-crosslinked corneas, immediately (A) and 24 hours (B) after treatment. Data are averages of $16 \mathrm{CXL}$ and 8 non-CXL. Error bars represent SD.

decreased similarly with the IOP increase in cross-linked eyes in both the anterior and the posterior corneal surfaces $(-309$ $\mu \mathrm{m}$ [anterior] and $-319 \mu \mathrm{m}$ [posterior] at $40 \mathrm{~mm} \mathrm{Hg} \mathrm{IOP)}$, whereas control eyes showed an increase in radius of curvature with the IOP increase (anterior by $221 \mu \mathrm{m}$ ) or remained constant [posterior]. With decreasing IOP, the anterior cornea nearly returned to its initial curvature $(-113 \mu \mathrm{m}[\mathrm{CXL}], 39.6$ $\mu \mathrm{m}$ [non-cross-linked]), whereas the posterior cornea remained practically constantly flattened $(-180 \mu \mathrm{m}[\mathrm{CXL}],-220$ $\mu \mathrm{m}$ [untreated]).
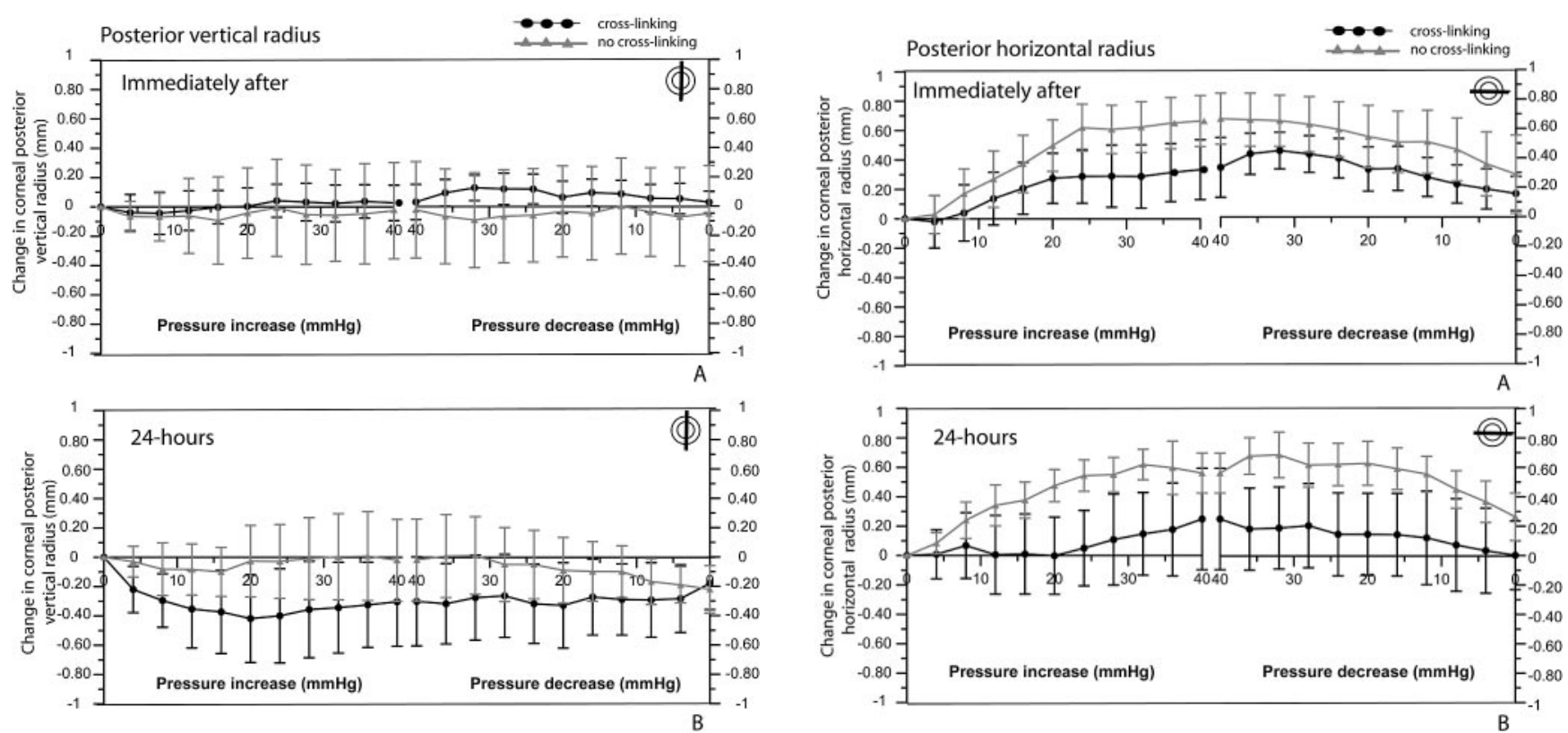

FIGURE 6. Change in posterior vertical radius of curvature with varying pressure (relative to $18.5 \mathrm{~mm} \mathrm{Hg}$ ) for cross-linked and non-crosslinked corneas, immediately (A) and 24 hours (B) after treatment. Data are average of $16 \mathrm{CXL}$ and 8 non-CXL. Error bars represent SD.

FIgURE 7. Change in anterior horizontal radius of curvature with varying pressure (relative to $18.5 \mathrm{~mm} \mathrm{Hg}$ ) for cross-linked and noncross-linked corneas, immediately (A) and 24 hours (B) after treatment. Data are average of $16 \mathrm{CXL}$ and 8 non-CXL. Error bars represent SD.

Changes in the horizontal corneal radius with IOP (Figs. 7 [anterior cornea] and 8 [posterior cornea]), as well as differences between the cross-linked and non-cross-linked corneas, were more dramatic than for the vertical radius. Figures $7 \mathrm{~A}$ and $8 \mathrm{~A}$ show the behavior immediately after treatment, and Figures $7 \mathrm{~B}$ and $8 \mathrm{~B}$ show the behavior 24 hours after treatment. In contrast to corneal thickness and vertical radius curvature, immediate measurements of horizontal radius curvature under IOP variation already showed larger changes in non-cross-

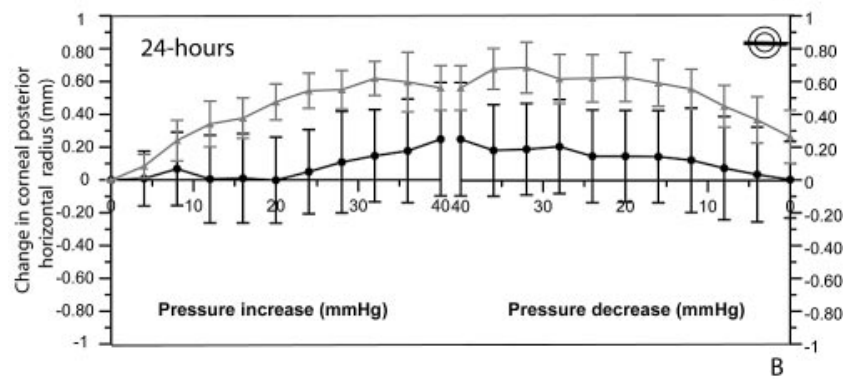

FIGURE 8. Change in posterior horizontal radius of curvature with varying pressure (relative to $18.5 \mathrm{~mm} \mathrm{Hg}$ ) for cross-linked and noncross-linked corneas, immediately (A) and 24 hours (B) after treatment. Data are average of $16 \mathrm{CXL}$ and 8 non-CXL. Error bars represent SD. 

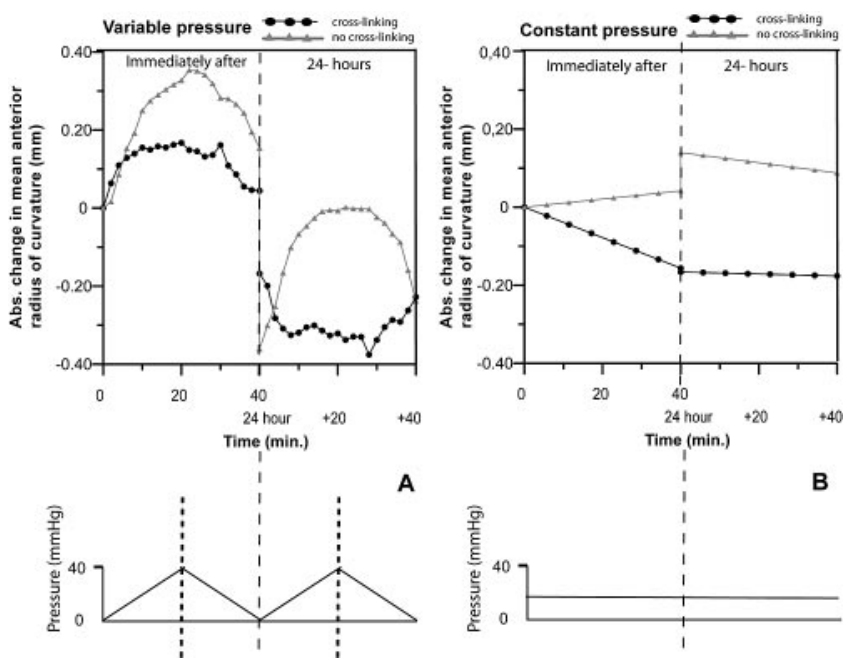

FIGURE 9. Change in anterior radius of curvature (averaged vertical and horizontal) as a function of time, relative to the value at the beginning of the experiment. (A) For variable pressure. (B) For constant pressure. The accompanying graphs represent the change of pressure (relative to $18.5 \mathrm{~mm} \mathrm{Hg}$ ) with time for cross-linked and non-cross-linked corneas. Pressure variations induced a characteristic corneal deformation (much larger in untreated than cross-linked corneas). Cross-linking also produced a significant decrease in corneal curvature (even under constant pressure).

linked corneas (768 $\mu \mathrm{m}$ [anterior], $665 \mu \mathrm{m}$ [posterior] at 40 $\mathrm{mm} \mathrm{Hg}$ IOP) than in cross-linked ones (398 $\mu \mathrm{m}$ [anterior], 448 $\mu \mathrm{m}$ [posterior] at $40 \mathrm{~mm} \mathrm{Hg} \mathrm{IOP})$. Measurements 24 hours after treatment showed the same tendency. Non-cross-linked corneas became significantly more deformed on IOP variation than cross-linked corneas $(P<0.01)$ Non- cross-linked corneas showed large increases in the horizontal radius of curvature with the IOP increase ( $504 \mu \mathrm{m}$ [anterior], $679 \mu \mathrm{m}$ [posterior] at $40 \mathrm{~mm} \mathrm{Hg}$ IOP) than did cross-linked corneas $(248 \mu \mathrm{m}$ [anterior] and $0 \mu \mathrm{m}$ [posterior]) and strong decreases with the IOP decrease. Non-cross-linked corneas experienced a plastic deformation because they did not return to their original radii of curvature (remaining $207 \mu \mathrm{m}$ [anterior] and $264 \mu \mathrm{m}$ [posterior] flatter) after IOP variation.

Changes in radius of curvature of non-cross-linked eyes were $5.9 \times$ higher in the horizontal than in the vertical meridian, whereas changes of cross-linked eyes were $4.3 \times$ higher in the vertical than in the horizontal meridian. This high difference between horizontal and vertical radii of curvature was confirmed by measurements by which the eye was turned $90^{\circ}$ within the holder, indicating that these meridional differences are inherent to the eye and unrelated to the experimental setup.

Control experiments showed that the changes in anterior and posterior horizontal radii of curvature were minimal under constant pressure throughout a session of the same duration. Changes in radius under constant pressure ranged between $-4.4 \mu \mathrm{m} / \mathrm{min}$ in cross-linked corneas immediately after treatment to $0.057 \mu \mathrm{m} / \mathrm{min}$ in cross-linked corneas 24 hours after treatment.

\section{Refractive Changes: Varying versus Constant Pressure}

The analysis in previous subsections was based on changes in corneal radius of curvature relative to the value of the radius of curvature at the beginning of the session. However, we observed consistent changes in the radius of curvature between the two sessions separated by 24 hours. Figure 9 shows the variation in anterior radius of curvature (arithmetic mean of horizontal and vertical radii), averaged across eyes, relative to the initial value of the experiment on day 1 in each eye. Figure 9A shows data with varying IOP (both immediately after and 24 hours after treatment). Figure 9B shows the data under constant IOP. Both conditions reveal that the cross-linking treatment produces a decrease in the corneal anterior radius of curvature (of $156 \mu \mathrm{m}$, from the data under constant IOP). This corneal steepening occurred immediately after treatment (at least during the 40 minutes of observation) and remained constant (also overnight). Under variable pressure, although the measurements in both sessions (immediately and 24 hours later) showed a similar pattern (large variations with IOP in non-cross-linked eyes and smaller variations in cross-linked eyes), there was a steepening from day 0 to day 1 .

\section{Simple Biomechanical Model: Young's Modulus and Hysteresis}

Figure 10 plots stress as a function of strain $(\sigma-\varepsilon)$ obtained using the theoretical computations for untreated and crosslinked eyes described (Figs. 10A [immediately after treatment], $10 \mathrm{~B}$ [after 24 hours]). Behavior with both the increasing and the decreasing pressure is represented. The slope of these curves in the linear region (first phase), for increased pressure, represented a measure of the Young's modulus of the corneathe steeper the curve, the stiffer the cornea. The increase in stiffness was clear immediately after the cross-link treatment. In addition, although there were some differences between the immediate and the 24-hour behavior, the main qualitative features remained. The major change after 24 hours occurred in the untreated cornea, most likely because of the effects of hydration. After 24 hours, the Young modulus in the crosslinked eyes $\left(1.096 \pm 0.30 \mathrm{kN} / \mathrm{m}^{2}\right)$ was significantly $(1.58 \times)$ higher than that of untreated eyes $\left(0.692 \pm 0.30 \mathrm{kN} / \mathrm{m}^{2}\right)$; therefore, cross-linking stiffened corneal tissue by $36.86 \%$.

The model also allowed us to evaluate the differences in the strain on increased or decreased stress. We calculated the area inside the curves presented in Figure 8. Although not directly a measure of hysteresis, this measure relates to the elastic behavior of the cornea. We found that the ratio of untreated/ cross-linked cornea stress-strain curve area was 12.8 immediately after treatment; 24 hours after treatment, it was 1.38 . Time (and probably differential effects of hydration) produced changes in opposite directions in the untreated and crosslinked corneas. We found that after decreasing pressure from $58 \mathrm{~mm} \mathrm{Hg}$ to the initial pressure, cross-linked corneas almost returned to their initial start points (strain, 0.002 [immediately] and 0.014 [24 hours later]), whereas control corneas showed a
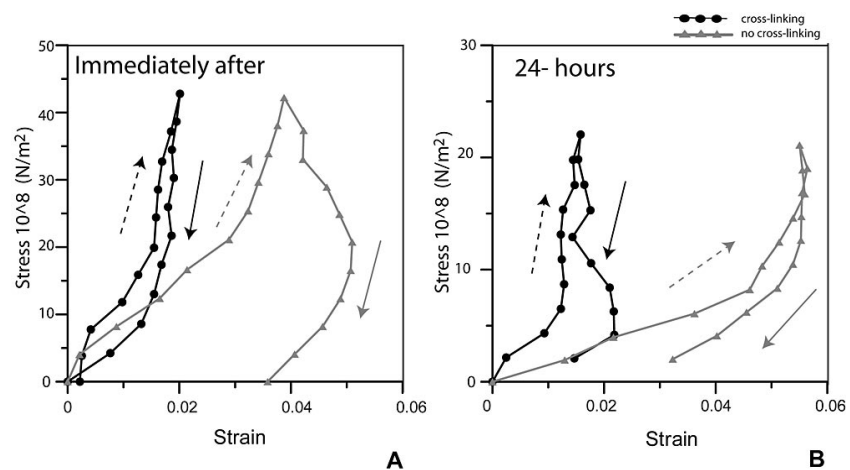

FIGURE 10. Strain-stress curves derived from the model of equation 1 for cross-linked and untreated corneas. Dashed arrows: increasing IOP; solid arrows: decreasing IOP. (A) Immediately and (B) 24 hours after treatment. 
large hysteresis and ended at higher strain (0.036 [immediately] and 0.032 (24 hours later). We conclude that crosslinked corneas were more elastic (although stiffness is higher) and did not show any remaining plastic deformations, in contrast to control eyes.

\section{Discussion}

\section{Comparison with Previous Clinical and Experimental Data}

The first major effect found during treatment is the decrease of corneal thickness after riboflavin instillation, probably caused by the hyperosmolarity of the riboflavin-dextran solution. We have observed that this has a strong dehydrating effect on the porcine cornea, which is reduced in thickness by $54 \%$. Decreased corneal thickness in patients has been reported recently in a clinical study ${ }^{31}$ in which corneal pachymetry was monitored with Scheimpflug imaging immediately after riboflavin instillation. On average, the corneal thickness decreased by $18.7 \%$, which may pose some safety concerns in thin corneas. Alternative hyposmolar riboflavin solution has been proposed to produce corneal swelling rather than dehydration to increase the effective corneal thickness. ${ }^{32}$ We observed an increase in corneal thickness caused by swelling 24 hours after treatment, as reported in previous studies on porcine corneas. ${ }^{7}$ However, we did not find that corneal hydration interfered with the corneal response to pressure variation 24 hours after treatment or that swelling effects were different between untreated and cross-linked corneas.

Even at constant pressure, we found a decrease in the corneal radius of curvature 24 hours after cross-linking (166 $\mu \mathrm{m})$. This is consistent with clinical reports of corneal steepening during the first month after treatment. ${ }^{33}$ After the initial steepening and 6 months after treatment, an average decrease in corneal curvature (flattening) of $2 \mathrm{D}$ and a decrease in refractive error of $1.14 \mathrm{D}^{34}$ have been reported clinically. Our results show effects of cross-linking immediately and 24 hours after treatment. The fact that refractive changes are observed in patients during the 6 months after treatment suggests would healing or long-term action balancing forces.

Previous inflation studies on excised porcine corneas showed a rise in the apex as a function of increased IOP very similar to our observations in untreated corneas (Fig. 4). As did Elsheiihk and Anderson, ${ }^{15}$ we found an initial phase $(0-24 \mathrm{~mm}$ $\mathrm{Hg}$ ) during which the corneal apex rises with pressure at a low stiffness (relatively high slope in Fig. 4), attributed to a relative looseness of the collagen fibers, followed by a much increased stiffness in the second phase, during which a significant increase in pressure results in a very small apical rise, attributed to the collagen fibers becoming taut. Previous studies used trephinate specimens (cornea and a narrow ring of scleral tissue) attached to a wet chamber rather than the whole globe used in the present study. The higher relative apical rise found in our study may result from height changes in the limbus rather than from the mechanical rigid attachment in the trephinate experiments. The limited lateral range of the Scheimpflug camera prevented us from accounting for the peripheral forward shift. Cross-linking reduced the apical rise by a factor of 2.8 in untreated corneas. Most notably, in cross-linked corneas, the second phase was reached at significantly lower loads (15 $\mathrm{mm} \mathrm{Hg}$ ), so that the regimen at physiological IOP was dominated by collagen with a much higher stiffness.

Although we cannot rule out a contribution of the entire eye motion to the apical radius displacement, the behavior we found in corneal apical shift was consistent with the changes in corneal thickness. Non-cross-linked corneas did not fully return to the initial values after increased and decreased IOP with regard to apical position or corneal thickness. Cross-linked corneas tended to return to the original values both for apical position (within the measurement variability) and for corneal thickness. These results are consistent with the cross-linked cornea being stiffer (but with a more elastic behavior) than the non-cross-linked cornea.

A previous study ${ }^{35}$ reported the decrease in central corneal thickness on globe inflation in two virgin human corneas in vitro using OCT (120 $\mu \mathrm{m}$ for a $206-\mathrm{mm} \mathrm{Hg}$ pressure increase). In that study, the posterior cornea was visible only in two corneas, and no data were provided for lower pressures. We found an average decrease of $73.6 \mu \mathrm{m}$ for an increase of $40 \mathrm{~mm}$ $\mathrm{Hg}$ in porcine corneas (average of 16 eyes). Cross-linking produced a reduction in the corneal thickness change by a factor of 2.28, consistent with an increase in corneal stiffness. The increase in the modulus of porcine corneal elasticity after CXL estimated from a simple model compares well with reports (a factor of $1.8 \times$ ) from corneal strip extensometry. ${ }^{14}$ The absolute values obtained from inflation tests are lower than those obtained from corneal strip extensometry.

We measured changes in anterior and posterior corneal radii of curvature as a function of IOP variation (Figs. 5-8). Changes in the posterior cornea tend to parallel the changes in the anterior cornea. We found highly significant differences between untreated and cross-linked corneas, consistent with an increase in corneal stiffness, particularly in the horizontal direction. We found high meridional differences both in the effect of IOP on corneal curvature and in the effect of the cross-linking treatment. In untreated corneas, an increase of 40 $\mathrm{mm} \mathrm{Hg}$ produced 8.26 times more increase in the horizontal than in the vertical anterior radius of curvature. The vertical posterior radius was nearly not altered by IOP variations, whereas the horizontal posterior radius changed by $500 \mu \mathrm{m}$ in the 0 - to $40-\mathrm{mm} \mathrm{Hg}$ range. This result is in contrast to previous corneal strip extensometry experiments on specimens cut along the vertical or horizontal meridians of fresh porcine corneas, which showed the same stress-strain responses along the vertical and horizontal directions and only marginally stiffer responses (2\%-8\%) diagonally. ${ }^{36}$ Microstructural studies of the fibrillar collagen structure in the porcine cornea show a primarily circumferential orientation. Other species, such as horses, cows, and marmosets, have a primarily vertical orientation in the collagen fibrils; in humans, the stromal fibrils have a preferential orientation in the superior-inferior and temporalnasal direction. ${ }^{37,38}$ The orientation-dependent corneal deformation found in our whole globe inflation experiments suggests that factors beyond the orientation of collagen fibrils play a role in the anisotropy of the biomechanical response.

Cross-linking was more effective in preventing an increase in anterior corneal radius (both anterior and posterior, immediately and 24 hours afterward) in the horizontal than in the vertical meridian. In fact, we found some vertical steepening with IOP in cross-linked corneas. The inherent astigmatism of the porcine eye (on average, the vertical meridian was $2.19 \%$ flatter than the vertical meridian) may play a role in the effective UV-irradiation treatment area (and, therefore, the relative exposure). However, differences of $710 \mu \mathrm{m}$ in the treatment area are not expected to result in these meridional differences in stiffness. Structural differences in the vertical and horizontal fibrils and a differential response to the formation of crosslinking bonds may be responsible for the observed meridional differences in the response to cross-linking.

\section{Implications of the Results}

We have provided experimental data of the change in apex shift, corneal thickness, and anterior and posterior corneal radii of curvature in a porcine eye inflation model after corneal 
CXL (and control eyes after epithelium removal and riboflavin instillation but not after UV-A irradiation). Although differences are expected on human corneas (particularly because of the relatively larger corneal cross-section treated) and in vivo, the complete experimental data set, along with estimations of the Young's modulus, are valuable input parameters in finite element models that will allow a better understanding and increased predictability of the cross-linking technique. To build a more sophisticated model with fewer limitations, we are planning further experiments with a high-resolution OCT and a larger lateral range that will allow viewing of limbal and scleral regions.

We have related some experimental findings on porcine corneas in vitro to clinical data found in patients. The use of a similar methodology on human corneal specimens, along with parameters obtained in vivo (particularly in eyes with corneal disorders susceptible to treatment) will provide further insight into the mechanism of cross-linking.

Open questions such as the apparent anisotropy in the biomechanical response of the porcine intact cornea and in response to treatment, yet to be confirmed in humans and of particular interest in pathologic corneas, require combined studies of microstructural analysis of the collagen fibers and biomechanical approaches.

\section{Acknowledgments}

The authors thank Daniel Pascual and Carlos Meneses for technical support and Michael Mrochen and Michael Büeler for initial discussions.

\section{References}

1. Wollensak G, Spoerl E, Seiler T. Riboflavin/ultraviolet-a-induced collagen crosslinking for the treatment of keratoconus. Am J Ophthalmol. 2003;135(5):620-627.

2. Wollensak G, Wilsch M, Spoerl E, Seiler T. Collagen fiber diameter in the rabbit cornea after collagen crosslinking by riboflavin/UV-A. Cornea. 2004;23(5):503-507.

3. Spoerl E, Huhle M, Seiler T. Induction of cross-links in corneal tissue Exp Eye Res. 1998;66(1):97-103.

4. Wollensak G, Spoerl E, Wilsch M, Seiler T. Endothelial cell damage after riboflavin-ultraviolet-A treatment in the rabbit. $J$ Cataract Refract Surg. 2003;29(9):1786-1790.

5. Wollensak G, Spoerl E, Wilsch M, Seiler T. Keratocyte apoptosis after corneal collagen cross-linking using riboflavin/UV-A treatment. Cornea. 2004;23(1):43-49.

6. Spoerl E, Mrochen M, Sliney D, Trokel S, Seiler T. Safety of UV-Ariboflavin cross-linking of the cornea. Cornea. 2007;26(4):385389.

7. Wollensak G, Aurich H, Pham DT, Wirbelbauer C. Hydration behavior of porcine cornea crosslinked with riboflavin and ultraviolet A. J Cataract Refract Surg. 2007;33(3):516-521.

8. Wollensak G. Crosslinkining treatment of progressive keratoconus: new hope. Curr Opin Ophthalmol. 2006;17(4):356-360.

9. Hafezi F, Kanellopoulos J, Wiltfang R, Seiler T. Corneal collagen crosslinking with riboflavin and ultraviolet $\mathrm{A}$ to treat induced keratectasia after laser in situ keratomileusis. J Cataract Refract Surg. 2007;33(12):2035-2040.

10. Ertan A, Colin J. Intracorneal rings for keratoconus and keratectasia. J Cataract Refract Surg. 2007;33(7):1303-1314.

11. Kohlhaas M, Spoerl E, Schilde T, Unger G, Wittig C, Pillunat LE. Biomechanical evidence of the distribution of cross-links in corneastreated with riboflavin and ultraviolet A light. J Cataract Refract Surg. 2006;32(2):279-283.

12. Wollensak G, Iomdina E. Long-term biomechanical properties of rabbit cornea after photodynamic collagen crosslinking. Acta Ophthalmol. 2009;87(1):48-51.
13. Spörl E, Schreiber J, Hellmund K, et al. Studies on the stabilization of the cornea in rabbits [in German]. Opbthalmologe. 2000;97(3): 203-206.

14. Wollensak G, Spoerl E, Seiler T. Stress-strain measurements of human and porcine corneas after riboflavin-ultraviolet-A-induced cross-linking. J Cataract Refract Surg. 2003;29(9):1780-1785.

15. Elsheikh A, Anderson K. Comparative study of corneal strip and extensometry and inflation tests. J R Soc Interface. 2005;2(3):177-185.

16. Pallikaris IG, Kymionis GD, Ginis HS, et al. Ocular rigidity in living human eyes. Invest Opbthalmol Vis Sci. 2005;46(2):409-414.

17. Asejczyk-Widlicka M, Pierscionek BK. The elasticity and rigidity of the outer coats of the eye. Br J Ophthalmol. 2008;92(10):1415-1418.

18. Jue B, Maurice DM. The mechanical properties of the rabbit and human cornea. J Biomech. 1986;19(10):847-853.

19. Hjortdal J. Regional elastic performance of the human cornea. J Biomech. 1996;29(7):931-942.

20. Anderson K, Elsheikh A, Newson T. Application of structural analysis to the mechanical behaviour of the cornea. $J R$ Soc Interface. 2004;1:3-15.

21. Boyce BL, Grazier JM, Jones RE, Nguyen TD. Full-field deformation of bovine cornea under constrained inflation conditions. Biomaterials. 2008;29:3896-3904.

22. Hennighausen H, Feldman ST, Bille JF, McCulloch AD. Anteriorposterior strain variation in normally hydrated and swollen rabbit cornea. Invest Ophthalmol Vis Sci. 1998;39(2):253-262.

23. Woo SL-Y, Kobayashi AS, Schlegel WA, Lawrence C. Nonlinear material properties of intact cornea and sclera. Exp Eye Res. 1972;14(1):29-39.

24. Shin TJ, Vito RP, Johnson LW, McCarey BE. The distribution of strain in the human cornea. J Biomech. 1997;30(5):497-503.

25. Srodka W, Iskander DR. Optically inspired biomechanical model of the human eyeball. J Biomed Opt. 2008;13(4):044034.

26. Elsheikh A, Alhasso D, Rama P. Biomechanical properties of human and porcine corneas. Exp Eye Res. 2008;86(5):783-790.

27. Zeng Y, Yang J, Huang K, Lee Z, Lee X. A comparison of biomechanical properties between human and porcine cornea. J Biomech. 2001;34(4):533-537.

28. Rosales P, Marcos S. Quantitative Scheimpflug imaging of the crystalline and intraocular lens. J Refract Surg. 2009;25(5):421-428.

29. Perez-Escudero A, Dorronsoro C, Sawides L, Remon L, MerayoLloves J, Marcos S. Minor influence of myopic laser in situ keratomileusis on the posterior corneal surface. Invest Ophthalmol Vis Sci. 2009;50(9):4146-4154.

30. Cano D, Barbero S, Marcos S. Comparison of real and computer-simulated outcomes of LASIK refractive surgery. JOSA A. 2004;21:926-936.

31. Kymionis GD, Kounis GA, Portaliou DM, et al. Intraoperative pachymetry measurements during corneal collagen cross-linking with riboflavin and ultraviolet A Irradiation. Ophthalmology. 2009; 116(12):2336-2339.

32. Hafezi F, Mrochen M, Iseli HP, Seiler T. Collagen crosslinking with ultraviolet-A and hypoosmolar riboflavin solution in thin corneas. $J$ Cataract Refract Surg. 2009;35(4):621-624.

33. Doors M, Tahzib NG, Eggink FA, Berendschot TTJM, Webers CAB, Nuijts RMMA. Use of anterior segment optical coherence tomography to study corneal changes after collagen cross-linking. $\mathrm{Am} \mathrm{J}$ Opbthalmol. 2009;148(6):844-851

34. Vinciguerra P, Albè E, Trazza S, et al. Refractive, topographic, tomographic and aberrometric analysis of keratoconic eyes undergoing corneal cross-linking. Ophthalmology. 2009;116(3):369-378.

35. Johnson CS, Mian SI, Moroi S, Epstein D, Izatt J, Afshari NA. Role of corneal elasticity in damping of intraocular pressure. Invest Opbthalmol Vis Sci. 2007;48(6):2540-2544.

36. Elsheikh A, Alhasso D. Mechanical anisotropy of porcine cornea and correlation with stromal microstructure. Exp Eye Res. 2009; 88(6): $1084-1091$

37. Hayes S, Boote C, Lewis J, et al. Comparative study of fibrillar collagen arrangement in the corneas of primates and other mammals. Anat Rec. 2007;290(12):1542-1550.

38. Meek KM, Newton R. Organization of collagen fibrils in the corneal stroma in relation to mechanical properties and surgical practice. J Refract Surg 1999;15(6):695-699. 